Results Among 25.005 participants, prevalence of daily and occasional household exposure was $12.5 \%$ and $21 \%$, respectively. Compared to non-exposed, daily household exposure decreased with increasing age, schooling and income. Occasional household exposure is not influenced by age, but decreases with increasing schooling and income. Compared to the Southeast Region, the most developed region in Brazil, daily exposure was lower in the North and Central West and higher in the Northeast. Among 10.933 participants with indoor occupations, $55 \%$ of men and $45 \%$ of women reported worksite exposure to passive smoking and $67 \%$ of them also reported household exposure. Prevalence of worksite exposure is higher in men, older adults (55+ years) and among participants with lower schooling and income and lower among South Region residents.

Conclusion Exposure to secondhand tobacco smoke, at home and at work, is very high and socially unequal in Brazil.

\section{SP4-15 BUILDING RESEARCH CAPACITY IN DEVELOPING COUNTRIES-COST EFFECTIVENESS OF AN EPIDEMIOLOGY COURSE TAUGHT BY TRADITIONAL AND VIDEO- TELECONFERENCING METHODS IN PAKISTAN}

doi:10.1136/jech.2011.142976p.9

${ }^{1} \mathrm{~S}$ Dodani, ${ }^{2} \mathrm{~T}$ Songer, ${ }^{2} \mathrm{R}$ LaPorte, ${ }^{3} \mathrm{Z}$ Ahmed. ${ }^{1}$ University of Kansas Medical center, Leawood, USA; ${ }^{2}$ University of Pittsburgh, Pittsburgh, USA; ${ }^{3}$ Unicom e-Health, Karachi, Pakistan

Building research capacity in developing countries using cost-effective methods has been recognised as an important pillar for the production of a sound evidence base for decision-making in policy and practice. We assessed the effectiveness of a research training course using traditional as well as video-teleconference method in Pakistan. A 9-day epidemiology research training course was offered to physicians in Pakistan (92\%) and Bangladeshis (8\%). The course was taught using (a) a traditional class room-face-to-face (F2F) method at the Aga Khan University, Karachi, Pakistan; and (b) Video Tele-Conferencing (VTC) method at two medical institutions within Pakistan. A total of 40 participants were selected for F2F and 46 for VTC group. Outcome parameters were assessed pre- and postcourse (short-term) as well as after 1 year (long-term). Costs of conducting the training in both methods were also identified using cost-effectiveness analysis.

Results The total study sample included 56 participants (F2F=38 and VTC=18) for the short-term and 49 participants for long-term assessment. After the end of the course (9th day), mean post-test1 scores showed significant improvement in both the groups that is, $15.08 \pm 1.75$ in F2F $(p=0.001)$ vs $13.122 \pm 1.87$ in VTC $(p=0.001)$ Mean scores after 1-year of the course (post-test 2) were lower than mean post-test 1 scores in both groups $(13.42 \pm 2.61$ in F2F vs $12.31 \pm 2.08$ in VTC), however, were higher than the baseline pretest scores.

Conclusion The use of e-technologies in developing countries proves to be an effective way of building capacity and reducing the problems of brain drain.

\section{SP4-16 DOES EDUCATION MODIFY THE ASSOCIATION BETWEEN SELF-RATED HEALTH AND MORTALITY AMONG OLDER PEOPLE IN INDONESIA?}

doi:10.1136/jech.2011.142976p.10
Reproductive Health, Faculty of Medicine, Gadjah Mada University, Yogyakarta, Indonesia

Introduction Evidences on whether poor self-reported health (SRH) predicts subsequent mortality across different socio-economic groups are inconsistent. This study assesses whether education modifies how poor SRH influences mortality among older people in Indonesia.

Methods A cohort of 11753 men and women aged 50 years and over was recruited in the INDEPTH/WHO Study on Adult Health and Global Ageing (SAGE) in Purworejo Health and Demographic Surveillance (HDSS) site in 2007. SRH was measured using the single global SRH question with 5-point response scales (very good, good, moderate $=$ moderately good SRH; bad, very bad=poor SRH) The baseline data were linked to the HDSS mortality data in 2010 HR for mortality was calculated for poor SRH using Cox proportional hazard regression after adjustment for age, education levels, age, marital status, living area, history of chronic diseases, and presence of disabilities.

Results During follow-up (median duration=37 months), 1199 deaths $(10.2 \%)$ and $1.9 \%$ lost to follow-up were identified. Poor SRH increased the mortality risk in men (HR 3.59, 95\% CI 1.96 to 6.57) and women (HR 3.16, 1.12 to 8.90). Education levels were not associated with mortality risk. The association between poor SRH and mortality did not differ across education groups, neither in men nor in women. Presence of disabilities, history of chronic diseases, and living alone increased the mortality risk.

Conclusion Poor SRH predicts mortality among older population in Indonesia. Education does not modify the association between poor $\mathrm{SRH}$ and mortality. Health promotion in the general population is important for the reduction of the mortality risk among older people.

\section{SP4-17 PREVALENCE OF HEPATITIS B AND C AMONG PREOPERATIVE CATARACT PATIENTS OF KARACHI}

doi:10.1136/jech.2011.142976p.11

E U Siddiqui, ${ }^{*}$ S S Naeem. Dow University of Health Sciences, Karachi, Sind, Pakistan

Introduction Hepatitis B and C are among the leading causes of morbidity and mortality, worldwide as well as in Pakistan. We intended to find out the prevalence of hepatitis B and C among preoperative cataract patients in Karachi. It is hypothesised that a high proportion of patients undergoing elective cataract surgery are infected with hepatitis B and C. Thus, it is imperative to come up with frequency to design strategies to decrease the burden.

Methods A descriptive study was conducted among 240 patients presenting for cataract surgery to Civil Hospital, Karachi. Diagnosis were made on the criteria that a patient must be positive for either $\mathrm{HBsAg}$ or Anti-HCV, or both. Convenience sampling was done after getting written informed consent.

Results Overall, five out of 235 (2.13\%) patients were HBsAg positive and 29 out of 239 (12.13\%) were Anti-HCV positive. One patient had a co-infection with both HBsAg and Anti-HCV positive. Conclusion High proportion of Hepatitis B and C are reported among preoperative cataract patients of Karachi. Routine serological screening prior to surgery should be made mandatory so that standard precautions could be taken and asymptomatic carrier patients would no longer pose a threat to its spread.

\section{SP4-18 A NEED ASSESSMENT STUDY OF HIV VOLUNTARY COUNSELLING AND TESTING SERVICES IN BANGLADESH}

doi:10.1136/jech.2011.142976p.12

S S Chowdhury, ${ }^{*}$ U Kulsum, S H Talukder. Eminence, Dhaka, Bangladesh

Backgrounds VCT is considered as an entry point for prevention and care and is acknowledged internationally as an effective strategy for 
both HIV/AIDS prevention and care. Bangladesh currently has 102 VCT centres. This study was aimed to identify the status of existing VCT services, analyse the policy documents and identify the needs perceived by the service recipients.

Methods 30 in depth interviews with key informants (eg, GoB, NAC, UNAIDS, WHO, INGOs) and 24 focus group discussion (FGDs) with 120 males and 97 females were conducted. The respondents include sex workers (M\&F), MSMs, transgender, PLWHIV, migrant workers and youth.

Results Existing VCT services are rather peer driven than voluntary. Neither the policy documents nor the existing intervention takes into account the needs of street children, underaged sex workers, adolescents and youth, leading to unequal VCT service coverage. Stigma and discrimination is the main reason for not seeking services. National policy or existing VCT guidelines do not suggest any unique mechanism for demand creating campaign.

Conclusions Policy reformulation and separate SOP for VCT is needed immediately. Streamlining of VCT services under government ownership can be an initiative for enhancing the service delivery regime. Health providers with excellent counselling skills are required in large numbers.

\section{SP4-19 USE OF LOGISTIC REGRESSION AND RECEIVER OPERATING CHARACTERISTIC CURVES TO DISCRIMINATE BETWEEN BLOOD DONORS AND NON-DONORS AMONG NORTH AMERICAN MEDICAL STUDENTS IN GRENADA, WEST INDIES}

doi:10.1136/jech.2011.142976p.13 been expressed regarding the continued rise of HIV infections among specific cultural groupings.

Objective To describe the association between socio-cultural practices (circumcision and pubic hair removal) and HIV infection risk in informal urban settlements.

Methods Data were collected from the Nairobi Urban Health and Demographic Surveillance System between January 2007 and December 2008. A total of 4767 residents of Viwandani and Korogocho slums, between the ages of 15-54 years for males and 15-49 years for females were recruited. Data were collected using interviewer-administered questionnaires. HIV serostatus was assessed using DetermineÒ HIV-1/HIV-2 (Abbott) and Uni-Gold Test kits.

Findings The highest HIV infection burden was observed among participants aged 25-34 years (40\%). Among HIV+ men, 64\% were circumcised compared to $88 \%$ among HIV - men. Majority of participants were circumcised before they were 12 years old and there were no differences among the HIV + and the HIV-. A similar proportion of HIV + (85\%) and HIV- $(83 \%)$ individuals had ever removed/shaved their pubic hair. After controlling for certain factors, circumcised men had a threefold reduced risk of developing HIV (OR 0.28; 95\% CI 0.16 to $0.47 ; p<0.01$ ) whereas those that had ever removed their pubic hair had only a onefold reduced risk of developing HIV (OR 0.98; 95\% CI $0.48 \%$ to $1.98 \%$; p $>0.05$ ).

Conclusion Our study found an association between circumcision and HIV, supporting findings from other studies.

\section{SP4-21 CLINICAL VALIDATION OF ANEROID SPHYGMOMANOMETER}

doi:10.1136/jech.2011.142976p.15

${ }^{1}$ L Messam, ${ }^{*}$ A Gomez, ${ }^{3}$ L Toner. 'University of California Davis, Davis, California, USA; ${ }^{2}$ Transglocal, Miami, Florida, USA; ${ }^{3}$ St. Georges University, True Blue, St. Georges, Grenada

Introduction The WHO estimates that 50 units of blood per 1000 inhabitants per year is necessary to meet the transfusion needs of a country. In Grenada 8.7 units are donated per 1000 inhabitants yearly. Methods We surveyed 452 St. George's University second year medical school students (>95\% North American) regarding blood donation attitudes and experiences before and after arrival in Grenada. This analysis of the 363 responses, determined if age, gender, knowledge of blood group, time since first arrival in Grenada and donating blood in the year preceding arrival would predict who donated (14\%) since arrival. In SPSS, we used logistic regression to estimate probabilities of donating blood since arrival as a function of each covariate. From these probabilities we generated Receiver Operating Characteristic curves with the area under each curve estimating the covariate's ability to predict blood donation after arrival in Grenada.

Results $68 \%$ of the time [95\% CI $60 \%$ to $76 \%$ ] students who had donated blood the year before arrival were more likely to be donors after arrival than those who had not. This percentage was slightly higher $(72 \%, 95 \%$ CI $63 \%$ to $80 \%)$ if, compared to the same group, these students had been in Grenada for a longer time and knew their blood group. Neither gender nor age showed predictive ability.

Conclusion Blood drive promotion should first target students who were not donors the year before arriving in Grenada as they are less likely to donate, without prompting, upon arrival.

\section{SP4-20 INFLUENCE OF SOCIO-CULTURAL PRACTICES ON HIV INFECTION IN TWO NAIROBI SLUMS; A CROSS-SECTIONAL STUDY}

doi:10.1136/jech.2011.142976p.14

S Mohamed, ${ }^{*} \mathrm{~N}$ Yatich, C Kyobutungi, A Ezeh. African Population Health \& Health Research Center, Nairobi, Kenya

Background HIV/AIDS is the leading cause of death in Africa accounting for more than $20 \%$ of all deaths. Major concerns have
${ }^{1}$ S S C Chan, ${ }^{*}{ }^{1} Z$ Z Wan, ${ }^{1} \mathrm{D}$ Y P Leung, ${ }^{2}$ A S M Abdullah, ${ }^{1} \mathrm{~A}$ J Hedley, ${ }^{1} \mathrm{~T}$ H Lam. ${ }^{1}$ The University of Hong Kong, Hong Kong, Hong Kong, ${ }^{2}$ Boston University, Boston, USA

Introduction Evidence had shown that nicotine replacement therapy (NRT) is effective to help smokers quit. Many studies reported a 